\title{
USING ADDITIVE FUNCTIONALS TO EMBED PREASSIGNED DISTRIBUTIONS IN SYMMETRIC STABLE PROCESSES $\left({ }^{1}\right)$
}

\author{
BY \\ ITREL MONROE
}

\begin{abstract}
Following Skorokhod, several authors in recent years have proposed methods to define a stopping time $T$ for Brownian motion $\left(X_{t}, \mathscr{F}_{t}\right)$ such that $X_{T}$ will have some preassigned distribution. In this paper a method utilizing additive functionals is explored. It is applicable not only to Brownian motion but all symmetric stable processes of index $\alpha>1$. Using this method one is able to obtain any distribution having a finite $\alpha-1$ absolute moment. There is also a discussion of the problem of approximating symmetric stable processes with random walks.
\end{abstract}

Introduction. Several authors in recent years have proposed methods to solve the following problem. Given a random variable $Y$ and a Brownian motion process $\left(X_{t}, \mathscr{F}_{t}\right)$ find a stopping time $T$ for $\left(X_{t}, \mathscr{F}_{t}\right)$ such that $X_{T}$ had the same distribution as $Y$. Skorokhod [11] first dealt with the problem in the course of showing that Brownian motion could be approximated by certain random walks. However, the stopping times that he used were measurable with respect to $\sigma$-algebras larger than those generated by the process itself. Root [10], while extending the work of Skorokhod and Dubins [4], used different methods to define the stopping times, both of which depended only on the sample paths.

In this paper a fourth method will be explored. The stopping times will depend on weighted combinations of local imes and are applicable not only to Brownian motion but also to symmetric stable processes of index $\alpha>1$. It will be shown that, using this method with a symmetric stable process of index $\alpha>1$, any distribution with an absolute moment of order $\alpha-1$ can be obtained (Theorem 16). In the case of Brownian motion, if the first moment of the distributions is zero, then the stopping time can be chosen such that the expectation of the stopping time is equal to the second moment of the distribution (Proposition 17). Theorem 13 seems to be of some interest in itself. It asserts that, if $f(x)=|x|^{\alpha-1}$ and $\left(X_{t}, \mathscr{F}_{t}\right)$ is the symmetric stable process of index $\alpha>1$ killed when $X_{t}=0$, then $\left(f\left(X_{t}\right), \mathscr{F}_{t}\right)$ is a martingale.

Presented to the Society, October 25,1969 under the title Utilizing additive functionals to define stopping times to obtain preassigned distributions; received by the editors June 25, 1970.

AMS 1969 subject classifications. Primary 6062; Secondary 6030.

Key words and phrases. Symmetric stable processes, additive functionals, stopping times, martingales, preassigned distributions.

( ${ }^{1}$ ) The results presented in this paper are contained in the author's doctoral dissertation prepared at the University of Washington under the direction of Professor Robert Blumenthal.

Copyright (C) 1972, American Mathematical Society 
Let $R$ be the real numbers with the usual topology $\mathscr{T}$ and $\mathscr{B}$ be the Borel sets generated by $\mathscr{T}$. The closure of a subset $A$ of $R$ will be denoted by $\bar{A}$, its interior by $A^{\circ}$, and its complement by $A^{c}$.

The set of continuous functions on $R$ will be denoted by $\mathscr{C}$ and the continuous functions with compact support will be denoted by $\mathscr{C}_{K}$. In general, our notation and terminology will follow that of Blumenthal and Getoor [2].

A measure is said to be a Radon measure if it is finite on compact sets. A sequence of Radon measures $\mu_{n}$ will be said to converge weakly to a Radon measure $\mu \neq 0$ if for all $f \in \mathscr{C}_{K}$

$$
\int f d \mu_{n} \rightarrow \int f d \mu
$$

We note that this differs from the weak convergence usually used in probability theory. See for instance [1].

Let $X=\left(X_{t}, \mathscr{F}_{t}\right)$ be the symmetric stable process of index $\alpha$ for $1<\alpha \leqq 2$. The process $X_{t}(\omega)$ will also be denoted by $X(t, \omega)$. We denote the basic probability space by $\Omega$ and denote the probability measure associated with the process originating at $x \in R$ by $P^{x}$. For each $t \geqq 0$ there is a map $\theta_{t}: \Omega \rightarrow \Omega$ such that $X_{s} \circ \theta_{t}=X_{t+s}$.

For a Borel set $A$ of $R$ let $T_{A}$ denote the stopping time

$$
T_{A}=\inf \left\{t: t>0, X_{t} \in A\right\} .
$$

An additive functional of the process $X$ is a family of functions

such that

$$
\left\{A_{t}: t \geqq 0, A_{t}: \Omega \rightarrow[0, \infty]\right\}
$$

(a) almost surely $A_{t}$ is nondecreasing and right continuous, and $A_{0}=0$;

(b) for each $t, A_{t} \in \mathscr{F}_{t}$;

(c) for each $t$ and $s, A_{s+t}=A_{t}+A_{s} \circ \theta_{t}$ a.s.

We shall also write $A(t, \omega)$ or $A(t)$ for $A_{t}$.

One defines the support of an additive functional $A$ by

$$
\operatorname{Supp}(A)=\left\{x: P^{x}(R=0)=1\right\}
$$

where $R=\inf \left\{t: A_{t}>0\right\}$. The support of $A$ is closed in the fine topology, which for the symmetric stable processes of index $\alpha>1$ coincides with $\mathscr{T}$. Thus $\operatorname{Supp}(A)$ is closed. Also $T_{\operatorname{Supp}(A)}=R$ and $\{t: A(t+\varepsilon)-A(t)>0$ for all $\varepsilon>0\} \subset\left\{t: X_{t} \in \operatorname{Supp}(A)\right\}$ almost surely.

An additive functional $A$ of $\boldsymbol{X}$ is said to be perfect if there is a set $\Lambda \in \mathscr{F}$ such that $P^{x}(\Lambda)=1$ for all $x$ and, if $\omega \in \Lambda, A_{t+s}(\omega)=A_{t}(\omega)+A_{s} \circ \theta_{t}(\omega)$ for all $t$ and $s$. $A$ is said to be continuous if almost surely the function $t \rightarrow A_{t}$ is continuous.

A continuous additive functional $L(t, x, \omega)$ (or $L(t, x)$ ) of $X$ is a local time at $x$ if $\operatorname{Supp}(L(t, x))=\{x\}$. The symmetric stable processes of index $\alpha>1$ do indeed have local times at each $x$ and, moreover, $L(t, x)$ can be chosen in such a way that it perfect, it is jointly continuous in $t$ and $x$, and for any Borel subset $A$ of $R$

$$
\int_{0}^{t} I_{A}\left(X_{s}\right) d s=\int_{A} L(t, x) d x
$$


where $I_{A}$ is the indicator function of $A$. Note that this implies that for fixed $t$ the function of $x, L(t, x)$, has compact support.

For further information on additive functionals, local times, and Markov processes in general, we refer to [2].

Let $M$ be the set of Radon measures on $(R, \mathscr{B})$ which are not identically zero. For $\mu \in M$ define

$$
A^{\mu}(t, \omega)=\int L(t, x, \omega) \mu(d x)
$$

Since $L(t, x)$ has compact support and $\mu$ is finite on compact sets, $A^{\mu}(t, \omega)$ is finite for all $t$. The dominated convergence theorem then yields the fact that $A^{\mu}(t, \omega)$ is continuous in $t$. It is then easily verified that $A^{\mu}(t, \omega)$ is a perfect continuous additive functional. $A^{\mu}(t, \omega)$ will also be denoted $A^{\mu}(t), A_{t}^{\mu}$ or simply $A^{\mu}$.

Lemma 1. Supp $\left(A^{\mu}\right)=\operatorname{Supp}(\mu)$.

Proof. First choose $z \in \operatorname{Supp}(\mu)$. Observe that since $\operatorname{Supp}(L(t, z))=\{z\}$,

$$
P^{z}(L(t, z)>0)=1
$$

for any $t>0$. Fix $t>0$. Using the continuity of $L(t, z)$ in $z$, there exists $P^{z}$ a.s. a neighborhood $U(\omega)$ of $z$ such that $L(t, x, \omega)>0$ on $U(\omega)$. Since $z \in \operatorname{Supp}(\mu)$, $\mu(U(\omega))>0$ so

$$
A^{\mu}(t, \omega)=\int L(t, x, \omega) \mu(d x) \geqq \int_{U(\omega)} L(t, x, \omega) \mu(d x)>0 \quad P^{z} \text { a.s. }
$$

Thus $P^{z}\left[\inf \left\{t: A_{t}^{\mu}>0\right\}=0\right]=1$ so $z \in \operatorname{Supp}\left(A^{\mu}\right)$.

Now consider a $z \notin \operatorname{Supp}(\mu)$. If $T=T_{\operatorname{Supp}(\mu)}$ then $P^{z}(T>0)=1$. Thus we can show that $z \notin \operatorname{Supp}\left(A^{\mu}\right)$ by showing that $A^{\mu}(T(\omega), \omega)=0 P^{z}$ a.s. To show that $A^{\mu}(T(\omega), \omega)$ $=0 P^{z}$ a.s. it suffices to show that $P^{z}$ a.s. $L(T(\omega), x, \omega)=0$ for all $x \in \operatorname{Supp}(\mu)$. Now for a given $x \in \operatorname{Supp}(\mu), L(T(\omega), x, \omega)=0 P^{z}$ a.s. since $T \leqq T_{\{x\}}$ and $\{x\}=$ $\operatorname{Supp}(L(t, x))$. Let $B$ be a countable dense subset of Supp $(\mu)$ and

$$
\Lambda=\{\omega: L(T(\omega), x, \omega)>0 \text { for some } x \in B\} .
$$

Clearly $P^{z}(\Lambda)=0$. Now, given any $x_{0} \in \operatorname{Supp}(\mu)$, if $L\left(T(\omega), x_{0}, \omega\right)>0$ then $L(T(\omega), x, \omega)>0$ for some $x \in B$, since $L(T(\omega), x, \omega)$ is continuous in $x$. Thus $\{\omega: L(T(\omega), x, \omega)=0$ for all $x \in \operatorname{Supp}(\mu)\} \subset\{\omega: L(T(\omega), x, \omega)=0$ for all $x \in B\}$ and the lemma follows.

LEMMA 2. If $E$ is a closed set then

$$
T_{E}=\inf \{t: L(t, x)>0 \text { for some } x \in E\} \quad \text { a.s. }
$$

Moreover for $t<T_{E}, E$ is contained in the interior of the set $\{x: L(t, x)=0\}$. 
Proof. Take $\mu$ to be a finite measure on $E$ such that $\operatorname{Supp}(\mu)=E$. Then, since Supp $\left(A^{\mu}\right)=E$, by 3.5 of Chapter V of [2]

$$
T_{E}=\inf \left\{t: \int L(t, x) \mu(d x)>0\right\} \text { a.s. }
$$

Using the continuity of $L(t, x)$ and the fact that $\operatorname{Supp}(\mu)=E$, one has

$$
\inf \{t: L(t, x)>0 \text { for some } x \in E\}=\inf \left\{t: \int L(t, x) \mu(d x)>0\right\}
$$

so the first statement is proved.

For the second statement, let $\left\{U_{n}\right\}$ be a decreasing sequence of open sets such that $E \subset U_{n} \subset \bar{U}_{n} \subset U_{n-1}$ so that $T_{n} \uparrow T_{E}$ where $T_{n}=T_{\bar{U}_{n}}$. If $t<T_{E}$ then $t<T_{n}$ for some $n$, so a.s. $L(t, x)=0$ for $x \in \bar{U}_{n}$ so the lemma is proved.

For $\mu \in M$ let $\tau^{\mu}(s)=\inf \left\{t: A_{t}^{\mu}>s\right\}$ and $\rho^{\mu}(s)=\inf \left\{t: A_{t}^{\mu} \geqq s\right\}$. One verifies that for fixed $s$ both $\tau^{\mu}(s)$ and $\rho^{\mu}(s)$ are stopping times. In addition $\tau^{\mu}(s)$ and $\rho^{\mu}(s)$ are finite a.s. Indeed for the symmetric stable processes $\lim _{t \rightarrow \infty} L(t, x)=\infty$ a.s. so $\lim _{t \rightarrow \infty} A_{t}^{\mu}=\infty$ a.s. which yields the finiteness of $\tau^{\mu}$ and $\rho^{\mu}$.

In the sequel we will drop the $\mu$ in the notation $A^{\mu}, \tau^{\mu}$, and $\rho^{\mu}$ when there is no danger of confusion.

Lemma 3. If $\mu \in M, X(\tau(s)) \in \operatorname{Supp}(A)$ a.s. Also for each $s, \tau(s)=\rho(s)$ a.s. and $\rho(s)=\lim _{u \uparrow s} \tau(u)$.

Proof. The last statement is obvious. To prove that $X(\tau(s)) \in \operatorname{Supp}(A)$, note that $A(\tau(s))<A(\tau(s)+\varepsilon)$ for any $\varepsilon>0$. Theorem 3.8 of Chapter $\mathrm{V}$ of [2] now implies that $X(\tau(s)) \in \operatorname{Supp}(A)$ a.s.

To show that $\tau(s)=\rho(s)$ a.s., recall first that the symmetric stable processes are quasi-left-continuous. That is, if $T_{n} \rightarrow T$ then $X\left(T_{n}\right) \rightarrow X(T)$ a.s. Choose an increasing sequence $u_{n} \rightarrow s$. Since $X\left(\tau\left(u_{n}\right)\right) \in \operatorname{Supp}(A)$ a.s., $X\left(\tau\left(u_{n}\right)\right) \rightarrow X(\rho(s))$ a.s. and $\operatorname{Supp}(A)$ is closed, we have $X(\rho(s)) \in \operatorname{Supp}(A)$ a.s. Now using the fact that $A(\rho(s))=s$ we have

$$
\begin{aligned}
E^{x}[\tau(s) & -\rho(s)] \\
& =E^{x}[\inf \{t: A(t)>s\}-\rho(s)]=E^{x}[\inf \{t: A(\rho(s)+t)>s\}] \\
& =E^{x}\left[\inf \left\{t: A(\rho(s))+A(t) \circ \theta_{\rho(s)}>s\right\}\right]=E^{x}\left[\inf \left\{t: A(t) \circ \theta_{\rho(s)}>0\right\}\right] \\
& =E^{x}\left[E^{X(\rho(s))}(\inf \{t: A(t)>0\})\right] .
\end{aligned}
$$

Since $X(\rho(s)) \in \operatorname{Supp}(A)$ a.s. and $\inf \{t: A(t)>0\}=0 P^{z}$ a.s. for $z \in \operatorname{Supp}(A)$, we have

$$
E^{x}[\tau(s)-\rho(s)]=E^{x}[0]=0 .
$$

Thus $\tau(s)=\rho(s)$ a.s.

PROPOSITION 4. If $\mu_{n}, \mu \in M$ and $\mu_{n} \rightarrow \mu$ weakly, then $X\left(\tau^{\mu_{n}}(s)\right) \rightarrow X\left(\tau^{\mu}(s)\right)$ a.s. for all $s \geqq 0$. 
Proof. Let $\tau_{n}=\tau^{\mu_{n}}$ and $A^{n}=A^{\mu_{n}}$. Since $L(t, x)$ is continuous in $x$ and has compact support a.s., $A_{t}^{n}=\int L(t, x) \mu_{n}(d x) \rightarrow \int L(t, x) \mu(d x)=A_{t}^{\mu}$ a.s. for all $t$. In particular

so

$$
A^{\mu}\left(\lim \inf \tau_{n}(s)\right)=s=A^{\mu}\left(\lim \sup \tau_{n}(s)\right)
$$

$$
\rho^{\mu}(s) \leqq \lim \inf \tau_{n}(s) \leqq \lim \sup \tau_{n}(s) \leqq \tau^{\mu}(s) .
$$

But $\rho^{\mu}(s)=\tau^{\mu}(s)$ a.s. so $\tau_{n}(s) \rightarrow \tau^{\mu}(s)$ a.s. The quasi-left-continuity of the process now yields the fact that a.s. $X\left(\tau_{n}(s)\right) \rightarrow X\left(\tau^{\mu}(s)\right)$ as asserted.

Although we could develop the following material with any fixed $s$, we will henceforth take $s=1$ so that $\tau^{\mu}=\inf \left\{t: A_{t}^{\mu}>1\right\}$.

We now come to the basic definition. For $\mu \in M$ define the measure $\psi(\mu)$ by

$$
\psi(\mu)(E)=P^{0}\left\{X\left(\tau^{\mu}\right) \in E\right\}
$$

for $E \in \mathscr{B}$.

We now give to $M$ the topology of weak convergence. In addition, we let $P$ be the set of probabilities on $(R, \mathscr{B})$ and give to $P$ the topology of weak convergence also. The next proposition gives two nice properties of the mapping $\psi$.

Proposition 5. If $\mu_{n} \rightarrow \mu$ weakly then $\psi\left(\mu_{n}\right) \rightarrow \psi(\mu)$ weakly and, moreover, $\operatorname{Supp}(\psi(\mu)) \subset \operatorname{Supp}(\mu)$.

Proof. By Proposition 1, if $\mu_{n} \rightarrow \mu$ weakly then $X\left(\tau^{\mu_{n}}\right) \rightarrow X\left(\tau^{\mu}\right)$ a.s., so in particular $\psi\left(\mu_{n}\right) \rightarrow \psi(\mu)$ weakly. By Lemma 1 , Supp $(\mu)=\operatorname{Supp}\left(A^{\mu}\right)$ and by Lemma $2 X(\tau) \in \operatorname{Supp}\left(A^{\mu}\right)$ a.s. Thus by the definition of $\psi$, Supp $(\psi(\mu)) \subset \operatorname{Supp}(\mu)$ a.s.

The next proposition can be found in [9].

Proposition 6. Let $S$ be a simplex, let $f: S \rightarrow S$ be continuous and suppose that $f$ maps each face of $S$ into itself. Then $f$ is onto.

Proposition 7. Let $E$ be a finite set and for $\alpha>0$

$$
M_{\alpha}^{E}=\{\mu \in M, \operatorname{Supp}(\mu) \subset E, \mu(E)=\alpha\} .
$$

Also let $P^{E}=\{\nu \in P$, Supp $(\nu) \subset E\}$. Then $\psi$ maps $M_{\alpha}^{E}$ onto $P^{E}$.

Proof. Let $E=\left\{a_{0}, a_{1}, \ldots, a_{k}\right\}$ and $S$ be a $k$-simplex with vertices $\left\{v_{0}, v_{1}, \ldots, v_{k}\right\}$. Define $h: M_{\alpha}^{E} \rightarrow S$ by

$$
h(\mu)=\left(\frac{1}{\alpha}\right) \sum_{i=0}^{k} \mu\left(a_{i}\right) v_{i}
$$

Clearly $h$ is one-to-one and onto. Next note that when $\mu_{n}, \mu \in M_{\alpha}^{E}, \mu_{n} \rightarrow \mu$ weakly if and only if $\mu_{n}\left(a_{i}\right) \rightarrow \mu\left(a_{i}\right)$. Thus $h$ is a homeomorphism. In the same manner we can define $g: P^{E} \rightarrow S$ by

$$
g(\nu)=\sum_{i=0}^{k} \nu\left(a_{i}\right) v_{i}
$$


and $g$ is a homeomorphism. Consider the map $g \circ \psi \circ h^{-1}: S \rightarrow S$. It is certainly continuous and, since $\operatorname{Supp}(\psi(\mu)) \subset \operatorname{Supp}(\mu), g \circ \psi \circ h^{-1}$ takes each face of $S$ into itself. Thus $g \circ \psi \circ h^{-1}$ is onto, which is equivalent to the assertion that $\psi$ is onto.

THEOREM 8. If $\nu$ is any probability measure with compact support, then for any $0<\alpha<\infty$ there is a $\mu \in M$ such that $\mu(R)=\alpha$, $\operatorname{Supp}(\mu)=\operatorname{Supp}(\nu)$ and $\psi(\mu)=\nu$.

Proof. Let $\nu_{n} \in P$ be a sequence of probability measures converging weakly to $\nu$ such that, for each $n$, Supp $\left(\nu_{n}\right)$ is a finite set and $\operatorname{Supp}\left(v_{n}\right) \subset \operatorname{Supp}(\nu)$. By Proposition 3, there is a $\mu_{n}$ with $\operatorname{Supp}\left(\mu_{n}\right)=\operatorname{Supp}\left(\nu_{n}\right), \mu_{n}(R)=\alpha$, and $\psi\left(\mu_{n}\right)=\nu_{n}$. Now since $\mu_{n}(R)=\alpha$ for all $n$ and $\operatorname{Supp}\left(\mu_{n}\right) \subset \operatorname{Supp}(\nu)$, which is compact by assumption, there exists a subsequence $\mu_{n^{\prime}}$ of $\mu_{n}$ which converges weakly to a measure $\mu$, where $\mu(R)=\alpha$ and $\operatorname{Supp}(\mu) \subset \operatorname{Supp}(\nu)$. Since $\psi$ is continuous,

$$
\psi(\mu)=\psi\left(\lim \mu_{n^{\prime}}\right)=\lim \psi\left(\mu_{n^{\prime}}\right)=\lim \nu_{n^{\prime}}=\nu .
$$

By Proposition 2, Supp $(\nu) \subset \operatorname{Supp}(\mu)$ so the theorem is proved.

If the measure $\mu$ is not finite on compact sets, then $A_{t}^{\mu}$ is not necessarily an additive functional, since it may not be continuous on the right. However, $\tau^{\mu}$ will still be a stopping time. This enables us to extend the domain of $\psi$ to a larger class of measures.

Let $M^{*}$ be the set of nonzero measures $\mu$ on $R$ such that $\lim _{n} \mu([x-1 / n, x+1 / n])$ $=\mu(\{x\})$ for all $x \in R$.

This is no restriction if $\mu([x-1 / n, x+1 / n])$ is finite for some $n$, but it requires $\mu(\{x\})$ to be infinite if the measure of every neighborhood of $x$ is infinite.

If $\mu_{n}, \mu \in M^{*}$, then we will say $\mu_{n}$ converges to $\mu$ if, for any open set $U$,

$$
\lim \inf \mu_{n}(U) \geqq \mu(U)
$$

and, for any compact set $C, \lim \sup \mu_{n}(C) \leqq \mu(C)$.

Notice that this convergence agrees with weak convergence if $\mu_{n}$ and $\mu$ are Radon measures.

Proposition 9. Suppose $\mu_{n}, \mu \in M^{*}$ and let $E=\{x: \mu(x)=\infty\}$. Then $\mu_{n} \rightarrow \mu$ if and only if

(1) for all open sets $U, U \cap E \neq \varnothing \Rightarrow \mu_{n}(U) \rightarrow \infty$,

(2) for all $f \in \mathscr{C}_{K}$ such that $\operatorname{Supp}(f) \cap E=\varnothing, \int f d \mu_{n} \rightarrow \int f d \mu$.

Proof. Since $\mu \in M^{*}, E$ is closed. Let $F=\left\{x: x \in U \in \mathscr{T} \Rightarrow \lim \mu_{n}(U)=\infty\right\}$.

(a) Suppose $\mu_{n} \rightarrow \mu$. Let $x \in E$. If $x \in U$ then $\mu(U)=\infty$ so $\lim \inf \mu_{n}(U)=\infty$. Thus $x \in E \Rightarrow x \in F$. If $x \notin E$ then there is an $\varepsilon>0$ such that $\mu([x-\varepsilon, x+\varepsilon])<\infty$. Then $\lim \sup \mu_{n}([x-\varepsilon, x+\varepsilon])<\infty$ so $x \notin F$. Thus $E=F$.

If $f \in \mathscr{C}_{K}$ and $\operatorname{Supp}(f) \cap E=\varnothing$ then $\operatorname{Supp}(f) \subset U$, where $\lim \sup \mu_{n}(U)<\infty$. Indeed, every point not in $E$ has such a neighborhood, so compact sets do also. Since $\lim \sup \mu_{n}(U)<\infty, \mu(U)<\infty$ also. Thus $\mu_{n}$ and $\mu$ are finite measures on $U$ and it is well known that the conditions on $\mu_{n}$ and $\mu$ imply that $\int f d \mu_{n} \rightarrow \int f d \mu$. 
(b) Assume statements (1) and (2). If $U$ is an open set and $U \cap E \neq \varnothing$, then $\lim \inf \mu_{n}(U)=\infty$, so in particular $\lim \inf \mu_{n}(U) \geqq \mu(U)$. If $U \cap E=\varnothing$ then $\int f d \mu_{n}$ $\rightarrow \int f d \mu$ for $f \in \mathscr{C}_{K}$ and $\operatorname{Supp}(f) \subset U$. Now if $I_{U}$ is the indicator function of $U$, then $I_{U}=\lim f_{i}$ where $f_{i} \leqq I_{U}$ and $f_{i} \in \mathscr{C}_{K}$. Clearly $\operatorname{Supp}\left(f_{i}\right) \subset U$. By the monotone convergence theorem, we can choose $f=f_{i}$ such that $\mu(U)-\int f d \mu<\varepsilon$ if $\mu(U)<\infty$, or $\int f d \mu>n$ if $\mu(U)=\infty$. Since $\int f d \mu_{n} \rightarrow \int f d \mu$, we have

$$
\begin{aligned}
\lim \inf \mu_{n}(U) & \geqq \lim \int f d \mu_{n} \\
=\int f d \mu>\mu(U)-\varepsilon & \text { if } \mu(U)<\infty, \\
>n & \text { if } \mu(U)=\infty .
\end{aligned}
$$

Since $\varepsilon$ and $n$ were arbitrary, $\lim \inf \mu_{n}(U) \geqq \mu(U)$.

Let $C$ be a compact set. If $C \cap E \neq \varnothing, \mu(C)=\infty$ so $\lim \sup \mu_{n}(C) \leqq \mu(C)$. If $C \cap E=\varnothing$, then, as before, $C$ is contained in an open set $U$ such that lim $\sup \mu_{n}(U)$ $<\infty$ and thus $\mu(U)<\infty$ also. Again, if $I_{C}$ is the indicator function of $C$, then $I_{C}=\lim f_{i}$, where $f_{i} \in \mathscr{C}_{K}, \operatorname{Supp}\left(f_{i}\right) \subset U, f_{i} \geqq I_{C}$, and $f_{i} \leqq 1$. Thus by the dominated convergence theorem, we can choose $f=f_{i}$ such that $\int f d \mu-\int I_{C} d \mu<\varepsilon$. Thus

$$
\lim \sup \mu_{n}(C) \leqq \lim \int f d \mu_{n}=\int f d \mu<\mu(C)+\varepsilon
$$

so again $\lim \sup \mu_{n}(C) \leqq \mu(C)$.

Proposition 10. If $\mu_{n} \in M^{*}$ and $\lim \inf \mu_{n}(K)>0$ for some compact set $K$, then there exists a subsequence $\mu_{n^{\prime}}$ of $\mu_{n}$ and a measure $\mu \in M^{*}$ such that $\mu_{n^{\prime}} \rightarrow \mu$.

Proof. Throughout, all subsequences will be denoted by $\mu_{n^{\prime}}$ even though they will be continually modified to satisfy new requirements.

Let $U_{k}$ be a countable base for $\mathscr{T}$ and select $\mu_{n^{\prime}}$ such that for all $k$ either $\lim \mu_{n^{\prime}}\left(U_{k}\right)=\infty$ or $\lim \sup \mu_{n^{\prime}}\left(U_{k}\right)<\infty$. Let

$$
F=\left\{x: x \in U \Rightarrow \lim \mu_{n^{\prime}}(U)=\infty\right\} \text {. }
$$

Again, $F$ is closed, and if $f \in \mathscr{C}_{K}$ such that $\operatorname{Supp}(f) \subset E^{c}$, then

$$
\lim \sup \int f d \mu_{n^{\prime}}<\infty \text {. }
$$

Denote by $\mathscr{C}_{K}^{\prime}$ the functions $f \in \mathscr{C}_{K}$ such that $\operatorname{Supp}(f) \cap F=\varnothing$. Let $f_{k}, k=1,2, \ldots$, be a countable set from $\mathscr{C}_{K}^{\prime}$ which is dense in $\mathscr{C}_{K}^{\prime}$ in the uniform topology. Select the $\mu_{n^{\prime}}$ such that $\int f_{k} d \mu_{n^{\prime}}$ converges for all $f_{k}$ and let $L\left(f_{k}\right)=\lim \int f_{k} d \mu_{n^{\prime}}$. Clearly $L$ can be extended to all of $\mathscr{C}_{K}^{\prime}$ and, on $\mathscr{C}_{K}^{\prime}, L$ is linear and nonnegative. Thus by the Riesz Representation Theorem there is a $\mu$ on $F^{c}$ such that $L(f)=\int f d \mu$ for all $f \in \mathscr{C}_{K}^{\prime}$. Extend the definition of $\mu$ by defining

$$
\mu(\{x\})=\infty \quad \text { if } x \in F .
$$


Then, by Proposition 9, $\mu_{n^{\prime}} \rightarrow \mu$. Clearly $\mu(K)>0$, so $\mu$ is not trivial.

Proposition 11. If $\mu_{n}, \mu \in M^{*}$ and $\mu_{n} \rightarrow \mu$, then $\psi\left(\mu_{n}\right) \rightarrow \psi(\mu)$.

Proof. As before, we will show that $\tau^{\mu_{n}} \rightarrow \tau^{\mu}$ a.s. Denote $\tau^{\mu_{n}}$ by $\tau_{n}$. Let $E=$ $\{x: \mu(x)=\infty\}$. By Lemma 2, if $t>T_{E}$ then a.s. $L(t, x)>0$ for some $x \in E$ so that $A_{t}^{\mu}=\infty$ a.s. Thus $\tau^{\mu} \leqq T_{E}$ a.s. By the same reasoning, $\lim \sup \tau_{n} \leqq T_{E}$ a.s.

Let $U_{k}$ be an increasing sequence of open sets such that $\bar{U}_{k} \subset U_{k+1}, \bar{U}_{k}$ is compact and $E^{c}=\bigcup U_{k}$. Define $\xi_{k}(A)=\mu\left(A \cap U_{k}\right)$. Recall that $\rho^{\xi}=\inf \left\{t: A_{t}^{\xi}=1\right\} \leqq \tau^{\xi}$ and that for finite measures $\rho^{\xi}=\tau^{\xi}$ a.s. We wish to show that $\rho^{\mu}=\tau^{\mu}$ a.s. If $\rho^{\mu}=T_{E}$ there is nothing to prove, since $\tau^{\mu} \leqq T_{E}$. If $\rho^{\mu}(\omega)<T_{E}(\omega)$, then for fixed $\omega$, the support of the continuous function of $x, L(t, x, \omega)$, is contained in $U_{k}$ for some $k$. Let $\Omega_{k}=\left\{\omega: \operatorname{Supp}\left(L\left(\rho^{\mu}, x, \omega\right)\right) \subset U_{k}\right\}$. On $\Omega_{k}$,

$$
1=\int L\left(\rho^{\mu}, x\right) \mu(d x)=\int L\left(\rho^{\mu}, x\right) \xi_{k}(d x)
$$

So $\rho^{\mu}=\rho^{\xi_{k}}=\tau^{\xi_{k}} \geqq \tau^{\mu}$ a.s. Thus $\rho^{\mu}=\tau^{\mu}$ a.s.

Let $S=\lim \inf \tau_{n}$. If $S<t<T_{E}$ then $\operatorname{Supp}(L(t, x)) \cap E=\varnothing$ a.s. so

$$
\int L(t, x) \mu_{n}(d x) \rightarrow \int L(t, x) \mu(d x) .
$$

Now $\lim \sup \int L(t, x) \mu_{n}(d x) \geqq 1$ so $\int L(t, x) \mu(d x) \geqq 1$. That is, $\rho^{\mu} \leqq S$. But $\rho^{\mu}=\tau^{\mu}$ a.s. so $\tau^{\mu} \leqq \lim$ inf $\tau_{n}$ a.s. if lim inf $\tau_{n}<T_{E}$. On the other hand this conclusion clearly holds if $\lim$ inf $\tau_{n} \geqq T_{E}$ since $\tau^{\mu} \leqq T_{E}$.

If $\tau^{\mu}<t<T_{E}$ then $\operatorname{Supp}(L(t, x)) \cap E=\varnothing$ so

$$
\int L(t, x) \mu_{n}(d x) \rightarrow \int L(t, x) \mu(d x)>1,
$$

and it follows that $\lim \sup \tau_{n}<\tau^{\mu}$. Thus, if either $\lim \inf \tau_{n}<T_{E}$ or $\tau^{\mu}<T_{E}$, then

$$
\tau^{\mu}=\lim \inf \tau_{n}=\lim \sup \tau_{n} \text { a.s. }
$$

The only other possibility is that $\tau^{\mu}=\lim \tau_{n}=T_{E}$, so the proposition is proved.

PROPOSITION 12. If $\nu$ is a probability measure with compact support, then there is a measure $\mu \in M^{*}$ such that $\operatorname{Supp}(\mu)=\operatorname{Supp}(\nu), \mu(R)=\infty$ and $\psi(\mu)=\nu$.

Proof. By Theorem 8, for each $n$ there exists a $\mu_{n} \in M$ such that Supp $\left(\mu_{n}\right)$ $=\operatorname{Supp}(\nu), \mu_{n}(R)=n$ and $\psi\left(\mu_{n}\right)=\nu$. Clearly $\lim \inf \mu_{n}(\operatorname{Supp}(\nu))>0$ so, by Proposition 10 , there exists a subsequence $\mu_{n^{\prime}}$ of $\mu_{n}$ and $\mu \in M^{*}$ such that $\mu_{n^{\prime}} \rightarrow \mu$. By Proposition $11, \psi(\mu)=\nu$. It also follows that

$$
\mu(R-\operatorname{Supp}(\nu)) \leqq \lim \inf \mu_{n^{\prime}}(R-\operatorname{Supp}(\nu))=0
$$

so $\operatorname{Supp}(\mu)=\operatorname{Supp}(\nu)$. Also

$$
\mu(\operatorname{Supp}(\nu)) \geqq \lim \sup \mu_{n^{\prime}}(\operatorname{Supp}(\nu))=\infty
$$

so $\mu(\operatorname{Supp}(\nu))=\infty$. 
The preceding material can be extended to somewhat more general spaces but for the following results we have to invoke properties of the symmetric stable processes in a very crucial manner.

THEOREM 13. Let $\left(X_{t}, \mathscr{F}_{t}\right)$ be the symmetric stable process of index $\alpha>1$ and $S_{0}$ an $\mathscr{F}_{t}$ stopping time such that $E\left\{\left|X\left(S_{0}\right)\right|^{\alpha-1}\right\}<\infty$. Define $S_{\infty}=S_{0}+T_{\{0\}} \circ \theta\left(S_{0}\right)$ and, for $t \geqq 0, S_{t}=\left(S_{0}+t\right) \wedge S_{\infty}$. Let $\mathscr{G}_{t}=\mathscr{F}\left(S_{t}\right)$ and $Y_{t}=\left|X\left(S_{t}\right)\right|^{\alpha-1}$. Then $\left(Y_{t}, \mathscr{G}_{t}\right)$ is a positive right continuous martingale.

Proof. Denote $T_{\{0\}}$ by $T$. We first show that for any $x \in R, t>0$,

$$
E^{x}\left\{|X(t \wedge T)|^{\alpha-1}\right\}=|x|^{\alpha-1} .
$$

To this end, a sequence of functions, $h_{n}$, is defined as follows. Let

$$
h_{n}(0)=0 \text { and } h_{n}(n)=2 n^{\alpha-1} /\left(n^{\alpha-1}-|1-n|^{\alpha-1}+1\right) .
$$

For all other $x$, let $T_{n}=T_{\{0, n\}}=\inf \left\{t: X_{t} \in\{0, n\}\right\}$ and define $h_{n}(x)=E^{x}\left\{h_{n} \circ X\left(T_{n}\right)\right\}$ $=P^{x}\left\{X\left(T_{n}\right)=n\right\} h_{n}(n)$. Getoor [5] has shown that

$$
P^{x}\left\{X\left(T_{n}\right)=n\right\}=\left(n^{\alpha-1}-|x-n|^{\alpha-1}+|x|^{\alpha-1}\right) / 2 n^{\alpha-1}
$$

so

$$
h_{n}(x)=\left(n^{\alpha-1}-|x-n|^{\alpha-1}+|x|^{\alpha-1}\right) /\left(n^{\alpha-1}-|1-n|^{\alpha-1}+1\right) .
$$

(In particular $h_{n}(1)=1$.)

Now observe that for any stopping time $R \leqq T_{n}$ we have $T_{n}=R+T_{n} \circ \theta_{R}\left(T_{n}\right.$ is the hitting time of $\{0, n\})$ and for any $a, P^{a}\left\{T_{\{a\}}=0\right\}=1$ so

$$
\begin{aligned}
E^{x}\left\{h_{n}(X(R))\right\} & =E^{x}\left\{E^{X(R)}\left\{h_{n} \circ X\left(T_{n}\right)\right\}\right\} \\
& =E^{x}\left\{h_{n} \circ X\left(R+T_{n} \circ \theta_{R}\right)\right\}=E^{x}\left\{h_{n} \circ X\left(T_{n}\right)\right\}=h_{n}(x) .
\end{aligned}
$$

In particular $E^{x}\left\{h_{n} \circ X\left(t \wedge T_{n}\right)\right\}=h_{n}(x)$.

We will show that $h_{n}(x) \rightarrow|x|^{\alpha-1}$ and that for fixed $t>0$, the functions on $\Omega$, $h_{n} \circ X\left(t \wedge T_{n}\right)$, are uniformly integrable. Since $P\left\{T_{n}=T\right\} \rightarrow 1$ these will imply that

$$
E^{x}\left\{|X(t \wedge T)|^{\alpha-1}\right\}=|x|^{\alpha-1} .
$$

To show that $h_{n}(x) \rightarrow|x|^{\alpha-1}$ note that the function $f(z)=z^{\alpha-1}$ has slope $f^{\prime}(z)$ $=(\alpha-1) z^{\alpha-2}$ which $($ since $\alpha<2)$ converges to zero for large $z$. Thus $n^{\alpha-1}-|n-x|^{\alpha-1}$ $\rightarrow 0$ as $n \rightarrow \infty$ so

$$
h_{n}(x)=\frac{n^{\alpha-1}-|n-x|^{\alpha-1}+|x|^{\alpha-1}}{n^{\alpha-1}-|n-1|^{\alpha-1}+1} \rightarrow|x|^{\alpha-1} .
$$

To show that the functions $h_{n} \circ X\left(t \wedge T_{n}\right)$ are uniformly integrable we will show that $\left|h_{n}(x)\right|<2|x|^{\alpha-1}<2 x+2$. To see that this is enough, observe that $\left|X_{s}\right|, s \leqq t$, is a right continuous positive submartingale so that the functions $\left|X\left(t \wedge T_{n}\right)\right|$ are uniformly integrable (see T19, VI of [8]). Now note that $h_{n}(n)>0$ so it follows from the definition that $h_{n}(x) \geqq 0$ for all $x \in R$. Also since $1<\alpha<2$ we have

$$
n^{\alpha-1}-|n-1|^{\alpha-1} \geqq 0
$$


so $0 \leqq h_{n}(x) \leqq n^{\alpha-1}-|n-x|^{\alpha-1}+|x|^{\alpha-1}$. We must show that $n^{\alpha-1}-|n-x|^{\alpha-1}$ $\leqq|x|^{\alpha-1}$ or rather $n^{\alpha-1} \leqq|x|^{\alpha-1}+|n-x|^{\alpha-1}$. If $n \leqq x$ or $x \leqq 0$ then $n^{\alpha-1} \leqq|x|^{\alpha-1}$ or $n^{\alpha-1} \leqq|n-x|^{\alpha-1}$ so there is nothing to prove. If $n>x>0$ then the inequality becomes $y^{\alpha-1}+z^{\alpha-1} \geqq(y+z)^{\alpha-1}$ for $y, z>0$ which follows from the fact that $f(z)=z^{\alpha-1}$ is concave and $f(0)=0$.

We have now established that

$$
E^{x}\left\{|X(t \wedge T)|^{\alpha-1}\right\}=|x|^{\alpha-1} .
$$

This immediately gives us that

$$
\begin{aligned}
E\left\{\left|X\left(S_{t}\right)\right|^{\alpha-1} \mid \mathscr{F}\left(S_{0}\right)\right\} & =E\left\{\left|X\left(\left(S_{0}+t\right) \wedge S_{\infty}\right)\right|^{\alpha-1} \mid \mathscr{F}\left(S_{0}\right)\right\} \\
& =E\left\{\left|X\left(S_{0}+\left(t \wedge T_{\{0\}}\right) \circ \theta\left(S_{0}\right)\right)\right|^{\alpha-1} \mid \mathscr{F}\left(S_{0}\right)\right\} \\
& =E^{X\left(S_{0}\right)}\left\{\left|X\left(t \wedge T_{\{0\}}\right)\right|^{\alpha-1}\right\}=\left|X\left(S_{0}\right)\right|^{\alpha-1} .
\end{aligned}
$$

By letting $S_{0}=S_{t}$, one obtains

$$
E\left\{\left|X\left(S_{t+s}\right)\right|^{\alpha-1} \mid \mathscr{F}\left(S_{t}\right)\right\}=\left|X\left(S_{t}\right)\right|^{\alpha-1} .
$$

This is the martingale property. The process is clearly right continuous.

COROllaRY 14. If $S_{0}$ is an $\mathscr{F}_{t}$ stopping time such that $E\left\{\left|X\left(S_{0}\right)\right|^{\alpha-1}\right\}<\infty$, and $T_{1}, T_{2}$ are $\mathscr{F}_{t}$ stopping times such that $S_{0} \leqq T_{1} \leqq T_{2} \leqq S_{\infty}=S_{0}+T_{\{0\}} \circ \theta_{S_{0}}$, then

$$
E\left\{\left|X\left(T_{2}\right)\right|^{\alpha-1}\right\} \leqq E\left\{\left|X\left(T_{1}\right)\right|^{\alpha-1}\right\} .
$$

If $T_{2} \leqq\left(S_{0}+t_{0}\right) \wedge S_{\infty}$, then equality holds.

Proof. Let $R_{1}=T_{1}-S_{0} . R_{1}$ is a $\mathscr{G}_{t}$ stopping time since

$$
\left\{R_{1} \leqq t\right\}=\left\{T_{1}-S_{0} \leqq t\right\}=\left\{T_{1} \leqq S_{0}+t\right\}=\left\{T_{1} \leqq\left(S_{0}+t\right) \wedge S_{\infty}\right\} \in \mathscr{F}\left(S_{t}\right)=\mathscr{G}_{t} .
$$

Likewise $R_{2}=T_{2}-S_{0}$ is a $\mathscr{G}_{t}$ stopping time. Using the fact that $\left(Y_{t}, \mathscr{G}_{t}\right)$ is a positive right continuous martingale, we have (13, Chapter VI, [8]) $E\left\{Y\left(R_{1}\right)\right\} \geqq E\left\{Y\left(R_{2}\right)\right\}$. But $Y\left(R_{1}\right)=\left|X\left(\left(S_{0}+R_{1}\right) \wedge S_{\infty}\right)\right|^{\alpha-1}=\left|X\left(T_{1}\right)\right|^{\alpha-1}$ so

$$
E\left\{\left|X\left(T_{1}\right)\right|^{\alpha-1}\right\} \geqq E\left\{\left|X\left(T_{2}\right)\right|^{\alpha-1}\right\} .
$$

In the case $T_{2} \leqq\left(S_{0}+t_{0}\right) \wedge S_{\infty}, R_{2} \leqq t_{0}$, so, since the family $Y_{t}, t \leqq t_{0}$, is uniformly integrable, equality holds.

COROLlaRY 15. If $\left(X_{t}, \mathscr{F}_{t}\right)$ is the symmetric stable process of index $\alpha>1$, then $\left(\left|X_{t}\right|^{\alpha-1}, \mathscr{F}_{t}\right)$ is a right continuous submartingale. If $T_{n}=\inf \left\{t:\left|X_{t}\right|>n\right\}$ then $\left(\left|X\left(t \wedge T_{n}\right)\right|^{\alpha-1}, \mathscr{F}_{t}\right)$ is a uniformly integrable submartingale.

Proof. Clearly $\left|X_{t+s}\right|^{\alpha-1} \geqq\left|X\left((t+s) \wedge\left(T_{\{0\}} \circ \theta_{t}\right)\right)\right|^{\alpha-1}$, and by Corollary 14,

$$
E\left\{\left|X_{t}\right|^{\alpha-1}\right\}=E\left\{\left|X\left((t+s) \wedge\left(T_{\{0\}} \circ \theta_{t}\right)\right)\right|^{\alpha-1}\right\} .
$$

Thus $\left(\left|X_{t}\right|^{\alpha-1}, \mathscr{F}_{t}\right)$ is a submartingale. Since $t \wedge T_{n}$ is a bounded $\mathscr{F}_{t}$ stopping time, it follows easily that $\left(\left|X\left(t \wedge T_{n}\right)\right|^{\alpha-1}, \mathscr{F}_{t}\right)$ is also a submartingale. It only remains to be shown that it is uniformly integrable. But when $m>n$,

$$
E\left\{\left|X\left(t \wedge T_{n}\right)\right|^{\alpha-1} ;\left|X\left(t \wedge T_{n}\right)\right|>m\right\} \leqq E\left\{\left|X\left(T_{n}\right)\right|^{\alpha-1} ;\left|X\left(T_{n}\right)\right|>m\right\}
$$

and by (1.4) of [3] $E\left\{\left|X\left(T_{n}\right)\right|^{\alpha-1}\right\}<\infty$. The assertion then follows. 
In a private communication, McKean has shown that it is not necessary to use Theorem 13 to show that $\left|X_{t}\right|^{\alpha-1}$ is a submartingale. In particular, his proof does not require the hitting distribution on which the proof of Theorem 13 was built, so, it has more appeal. However it does not yield Theorem 13. It should also be mentioned that McKean proved a much more general version of Corollary 15 in the case $\alpha<1$ [7].

THEOREM 16. Let $\left(X_{t}, \mathscr{F}_{t}\right)$ be the symmetric stable process of index $1<\alpha \leqq 2$. If $\nu \in P$, then $\nu$ is in the range of $\psi$ if and only if $\int|x|^{\alpha-1} \nu(d x)<\infty$.

Proof. Suppose $\psi(\mu)=\nu$. Choose $t_{0}$ such that $P\left\{\tau^{\mu}<t_{0}\right\}=\rho>0$. We will show that

$$
\int|x|^{\alpha-1} \nu(d x)=E\left\{\left|X\left(\tau^{\mu}\right)\right|^{\alpha-1}\right\} \leqq(2 / \rho) E\left\{\left|X\left(t_{0}\right)\right|^{\alpha-1}\right\} .
$$

To this end, let $T_{n}=\inf \left\{t:\left|X_{t}\right|>n\right\}, \tau_{n}=T_{n} \wedge \tau^{\mu}$ and $\rho_{n}=P\left\{\tau_{n}<t_{0}\right\}$. Clearly, $\tau_{n} \rightarrow \tau^{\mu}$, so that by quasi-left-continuity $X\left(\tau_{n}\right) \rightarrow X\left(\tau^{\mu}\right)$ a.s., and by Fatou's lemma

$$
E\left\{\left|X\left(\tau^{\mu}\right)\right|^{\alpha-1}\right\} \leqq \lim \inf E\left\{\left|X\left(\tau_{n}\right)\right|^{\alpha-1}\right\} .
$$

Thus it will be enough to show that $E\left\{\left|X\left(\tau_{n}\right)\right|^{\alpha-1}\right\} \leqq(2 / \rho) E\left\{\left|X\left(t_{0}\right)\right|^{\alpha-1}\right\}$.

Now, let $S=\left(t_{0}+T_{\{0\}} \circ \theta\left(t_{0}\right)\right) \wedge T_{n}$. In words, $S$ is the first time the process escapes from the interval $[-n, n]$, or, hits zero after time $t_{0}$. Now write

$$
\begin{aligned}
E\left\{\left|X\left(\tau_{n}\right)\right|^{\alpha-1}\right\}= & E\left\{\left|X\left(\tau_{n}\right)\right|^{\alpha-1} ; \tau_{n}<t_{0}\right\} \\
& +E\left\{\left|X\left(\tau_{n}\right)\right|^{\alpha-1} ; t_{0} \leqq \tau_{n} \leqq S\right\}+E\left\{\left|X\left(\tau_{n}\right)\right|^{\alpha-1} ; \tau_{n}>S\right\} .
\end{aligned}
$$

For the first term on the right-hand side, we use the fact that $\left|X_{t}\right|^{\alpha-1}$ is a right continuous submartingale to obtain

$$
E\left\{\left|X\left(\tau_{n}\right)\right|^{\alpha-1} ; \tau_{n}<t_{0}\right\} \leqq E\left\{\left|X\left(\tau_{n} \wedge t_{0}\right)\right|^{\alpha-1}\right\} \leqq E\left\{\left|X\left(t_{0}\right)\right|^{\alpha-1}\right\} .
$$

For the second term we certainly have

$$
E\left\{\left|X\left(\tau_{n}\right)\right|^{\alpha-1} ; t_{0} \leqq \tau_{n} \leqq S\right\} \leqq E\left\{\left|X\left(t_{0} \vee\left(\tau_{n} \wedge S\right)\right)\right|^{\alpha-1}\right\}
$$

since $t_{0} \vee\left(\tau_{n} \wedge S\right)=\tau_{n}$ on $t_{0} \leqq \tau_{n} \leqq S$. But $t_{0} \leqq t_{0} \vee\left(\tau_{n} \wedge S\right) \leqq t_{0}+T_{\{0\}} \circ \theta\left(t_{0}\right)$ so by Corollary 14 this second term is bounded by $E\left\{\left|X\left(t_{0}\right)\right|^{\alpha-1}\right\}$.

For the third term, consider the following. If $\tau_{n}>S$, then $X(S)=0$. Thus the process is where it was to begin with except for the fact that $P\left\{\tau_{n}>S\right\} \leqq 1-\rho$ and $A^{\mu}(S)$ might be positive. However if $A^{\mu}(S)$ is positive then we would expect to stop sooner than if we were to start afresh so we anticipate that

$$
E\left\{\left|X\left(\tau_{n}\right)\right|^{\alpha-1} ; \tau_{n}>S\right\} \leqq(1-\rho) E\left\{\left|X\left(\tau_{n}\right)\right|^{\alpha-1}\right\} .
$$

This together with the above would lead us to the inequality

or

$$
E\left\{\left|X\left(\tau_{n}\right)\right|^{\alpha-1}\right\} \leqq 2 E\left\{\left|X\left(t_{0}\right)\right|^{\alpha-1}\right\}+(1-\rho) E\left\{\left|X\left(\tau_{n}\right)\right|^{\alpha-1}\right\}
$$

$$
E\left\{\left|X\left(\tau_{n}\right)\right|^{\alpha-1}\right\} \leqq(2 / \rho) E\left\{\left|X\left(t_{0}\right)\right|^{\alpha-1}\right\}
$$

which is the desired inequality. 
Let us verify that

$$
E\left\{\left|X\left(\tau_{n}\right)\right|^{\alpha-1} ; \tau_{n}>S\right\} \leqq(1-\rho) E\left\{\left|X\left(\tau_{n}\right)\right|^{\alpha-1}\right\} .
$$

Let $\tau_{n}^{*}=S+\tau_{n} \circ \theta_{S}$ so that $\tau_{n}$ is the stopping time one would obtain if one did start afresh at time $S$. One verifies that $\tau_{n}^{*} \leqq T_{n}$. Also $\tau_{n}^{*} \geqq \tau_{n}$. Indeed, if $\tau_{n}>S$ then

$$
\begin{aligned}
\tau^{\mu} \circ \theta_{S} & =\inf \left\{t: A_{t} \circ \theta_{S}>1\right\} \\
& \geqq \inf \left\{t: A_{S}+A_{t} \circ \theta_{S}>1\right\}=\inf \left\{t: A_{S+t}>1\right\}=\tau^{\mu}-S
\end{aligned}
$$

so $\tau_{n}^{*}=\left(S+\tau^{\mu} \circ \theta_{S}\right) \wedge T_{n} \geqq \tau^{\mu} \wedge T_{n}=\tau_{n}$. Now using the fact that $\left|X\left(t \wedge T_{n}\right)\right|^{\alpha-1}$ is a uniformly integrable submartingale, we obtain

$$
\begin{aligned}
E\left\{\left|X\left(\tau_{n}\right)\right|^{\alpha-1} ; \tau_{n}>S\right\} & \leqq E\left\{\left|X\left(\tau_{n}^{*}\right)\right|^{\alpha-1} ; \tau_{n}>S\right\}=E\left\{\left|X\left(S+\tau_{n} \circ \theta_{S}\right)\right|^{\alpha-1} ; \tau_{n}>S\right\} \\
& =E\left\{E^{X(S)}\left[\left|X\left(\tau_{n}\right)\right|^{\alpha-1}\right] ; \tau_{n}>S\right\}=E\left\{E^{0}\left[\left|X\left(\tau_{n}\right)\right|^{\alpha-1}\right] ; \tau_{n}>S\right\}
\end{aligned}
$$

since $X(S)=0$ if $\tau_{n}>S$. But $E^{0}\left\{\left|X\left(\tau_{n}\right)\right|^{\alpha-1}\right\}$ is constant, so

$$
E\left\{\left|X\left(\tau_{n}\right)\right|^{\alpha-1} ; \tau_{n}>S\right\} \leqq E\left\{\left|X\left(\tau_{n}\right)\right|^{\alpha-1}\right\} E\left\{\tau_{n}>S\right\} \leqq(1-\rho) E\left\{\left|X\left(\tau_{n}\right)\right|^{\alpha-1}\right\},
$$

and the inequality is demonstrated. Thus,

$$
\int|x|^{\alpha-1} \nu(d x) \leqq \frac{2}{\rho} E\left\{\left|X\left(t_{0}\right)\right|^{\alpha-1}\right\}
$$

which is finite since a stable distribution of index $\alpha$ has finite $\gamma$ absolute moments for all $\gamma<\alpha$. Thus half of the theorem is proved.

Now suppose that $\int|x|^{\alpha-1} \nu(d x)<M<\infty$. We know that if $\nu$ has compact support, then $\nu$ is in the range of $\psi$. We thus suppose that the support of $\nu$ is not compact and define a sequence of measures $\nu_{n}$ such that $\operatorname{Supp}\left(\nu_{n}\right)$ is compact and $\nu_{n}$ converges to $\nu$ weakly. In particular, let $\varepsilon_{n}$ and $\varepsilon_{-n}$ denote the probability measures with unit mass at $n$ and $-n$ respectively and let

$$
\nu_{n}(A)=\nu(A \cap(-n, n))+\lambda_{n} \varepsilon_{-n}(A)+\rho_{n} \varepsilon_{n}(A)
$$

where $\lambda_{n}=\nu(-\infty, n]$ and $\rho_{n}=\nu[n, \infty)$. By Proposition 12, there is a measure $\varepsilon_{n}$ such that $\psi\left(\mu_{n}\right)=\nu_{n}$ and $\mu_{n}(R)=\infty$. If the sequence $\left\{\mu_{n}\right\}$ has a subsequence which converges weakly to some measure $\mu$, then by Proposition $11, \psi(\mu)=\nu$ and we are done.

Assume that $\left\{\mu_{n}\right\}$ has no weakly convergent subsequence. By Proposition 10, this is equivalent to assuming that $\lim \mu_{n}(C)=0$ for every compact set $C$. We will show that this leads to a contradiction.

If $\mu_{n}(C) \rightarrow 0$ for all compact sets $C$, then since the function of $x, L(t, x, \omega)$, has compact support a.s., it follows that $A^{\mu_{n}}(t, \omega) \rightarrow 0$ a.s. for each $t>0$. This clearly implies that if $\tau_{n}=\inf \left\{t: A^{\mu}(t)>1\right\}$ then $\tau_{n} \rightarrow \infty$ a.s. In particular, for any fixed number $a$, we can choose $n$ large enough that $P\left\{\tau_{n}>T_{\{a\}}\right\}>\frac{1}{2}$ and $P\left\{\tau_{n}>T_{\{-a\}}\right\}>\frac{1}{2}$.

To obtain the desired contradiction, let $a=(4 M)^{1 /(\alpha-1)}$ where $M \geqq \int|x|^{\alpha-1} \nu(d x)$ $\geqq \int|x|^{\alpha-1} \nu_{n}(d x)$ and we also assume that $M \geqq 1$. 
Now $\mu_{n}(R)=\infty$ and $\mu_{n}$ has compact support, so there is a point $b$ such that $\mu_{n}(\{b\})=\infty$. Since $\tau_{n} \leqq T_{\{b\}}$ (see the proof of Proposition 11), it follows that $|b|>a$. If $b>0$, take $S_{0}=\tau_{n} \wedge T_{\{a\}}$. (The case $b<0$ can be treated similarly.) Let $T_{1}=\tau_{n} \wedge S_{\infty}$ where $S_{\infty}=S_{0}+T_{\{0\}} \circ \theta\left(S_{0}\right)$. Clearly $X\left(T_{1}\right)=X\left(\tau_{n}\right)$ unless $X\left(T_{1}\right)=0$ so

$$
E\left\{\left|X\left(\tau_{n}\right)\right|^{\alpha-1}\right\} \geqq E\left\{\left|X\left(T_{1}\right)\right|^{\alpha-1}\right\} .
$$

On the other hand if $T_{2}=S_{\infty} \wedge T_{\{b\}}$, then, since $\tau_{n} \leqq T_{\{b\}}, S_{0} \leqq T_{1} \leqq T_{2} \leqq S_{\infty}$ so, by Corollary 14,

$$
E\left\{\left|X\left(T_{1}\right)\right|^{\alpha-1}\right\} \geqq E\left\{\left|X\left(T_{2}\right)\right|^{\alpha-1}\right\} .
$$

Thus to obtain the contradiction, it is enough to show that $E\left\{\left|X\left(T_{2}\right)\right|^{\alpha-1}\right\}>M$.

Now we have chosen $n$ such that $P\left\{\tau_{n}>T_{\{a\}}\right\}>\frac{1}{2}$ so $P\left\{S_{0}=T_{\{a\}}\right\}>\frac{1}{2}$ and

$$
P\left\{X\left(S_{0}\right)=a\right\}>\frac{1}{2} \text {. }
$$

Now this implies that

$$
\begin{aligned}
P\left\{X\left(T_{2}\right)=b\right\} & =P\left\{T_{\{b\}}<S_{\infty}\right\}=P\left\{S_{0}+T_{\{b\}} \circ \theta\left(S_{0}\right)<S_{0}+T_{\{0\}} \circ \theta\left(S_{0}\right)\right\} \\
& =E\left\{P^{X\left(S_{0}\right)}\left\{T_{\{b\}}<T_{\{0\}}\right\}\right\} \geqq \frac{1}{2} P^{a}\left\{T_{\{b\}}<T_{\{0\}}\right\} .
\end{aligned}
$$

Using Corollary 6.8 of [5] we have

Thus

$$
P\left\{X\left(T_{2}\right)=b\right\} \geqq \frac{1}{2}\left[\left(b^{\alpha-1}+a^{\alpha-1}-(b-a)^{\alpha-1}\right) / 2 b^{\alpha-1}\right] .
$$

$$
E\left\{\left|X\left(T_{2}\right)\right|^{\alpha-1}\right\}>\frac{a^{\alpha-1}+\left[b^{\alpha-1}-(b-a)^{\alpha-1}\right]}{4 b^{\alpha-1}} \cdot b^{\alpha-1}>\frac{1}{4} a^{\alpha-1}
$$

since $b>a>0$ so that $b^{\alpha-1}-(b-a)^{\alpha-1}>0$. But $a^{\alpha-1}=4 M$ so $E\left\{\left|X\left(T_{2}\right)\right|^{\alpha-1}\right\}>M$. This is the desired contradiction so the theorem is proved.

[Note. In a recent paper, Loynes gives a partial answer to the question of what distributions can be obtained using the stopping times depending on barriers as defined by Root. Modifying the proof above, one can show that (for Brownian motion) using barriers one can obtain a given distribution if and only if it has a finite absolute moment.]

The interest in Theorem 16 results from its usefulness in showing that Brownian motion can be approximated by certain random walks. For this purpose it is important to know that the expectation of the stopping time is finite. Of course in general this cannot be true, but, for Brownian motion we can show that it is true in the situations where one expects it to be.

Proposition 17. Let $\left(X_{t}, \mathscr{F}_{t}\right)$ be Brownian motion and $Y$ be a random variable such that $E\{Y\}=0$ and $E\left\{Y^{2}\right\}<\infty$. Then, there is a measure $\mu$ such that $X\left(\tau^{\mu}\right)$ has the same distribution as $Y$ and $E\left\{\tau^{\mu}\right\}<\infty$.

Proof. Let $\nu$ be the measure induced by $Y$ on $R$. If Supp $(\nu)$ is compact, let $a=\inf \operatorname{Supp}(\nu)$ and $b=\sup \operatorname{Supp}(\nu)$. By Proposition 12, there is a measure $\alpha \in M^{*}$ with infinite mass such that $\psi(\alpha)=\nu$ and $\operatorname{Supp}(\alpha)=\operatorname{Supp}(\nu)$. Let $E=\{x: \alpha(x)=\infty\}$. 
Since $\int x d \nu=0$ we have $a \leqq 0 \leqq b$, and, since Brownian motion has continuous paths and $\tau^{\alpha} \leqq T_{E}$ (see the proof of Proposition 11), we must have $E \subset\{a, b\}$. We will show that $E$ can be taken to be $\{a, b\}$.

Of course $E \neq \varnothing$ so let us suppose that $E=\{a\}$. Let $T=T_{\{a, b\}} \wedge \tau^{\alpha}$. Then

$$
\left\{X(T) \neq X\left(\tau^{\alpha}\right)\right\} \subset\{X(T)=b\}
$$

so $X(T) \geqq X\left(\tau^{\alpha}\right)$ a.s. Using the martingale properties of Brownian motion, we have $E\left\{X_{T}\right\}=0$. But $E\left\{X\left(\tau^{\alpha}\right)\right\}=0$ also so $X(T)=X\left(\tau^{\alpha}\right)$ a.s. That is, $X\left(\tau^{\alpha}\right)=b$ on the set $\tau^{\alpha}>T_{\{b\}}$ a.s. Define $\mu \in M^{*}$ by $\mu(b)=\infty$ and $\mu(A)=\alpha(A)$ if $A \cap\{b\}=\varnothing$. Then $\tau^{\mu}=\tau^{\alpha}$ unless $\tau^{\alpha}>T_{\{b\}}$. But in that case $X\left(\tau^{\mu}\right)=b=X\left(\tau^{\alpha}\right)$ so $X\left(\tau^{\mu}\right)$ and $X\left(\tau^{\alpha}\right)$ have the same distribution, the distribution of $Y$. Also $\tau^{\mu} \leqq T_{\{a, b\}}$ so $E\left\{\tau^{\mu}\right\}<\infty$.

Now suppose that Supp $(\nu)$ is not compact and choose a sequence of probability measures $v_{n}$ with compact support such that $\nu_{n} \rightarrow \nu, \int x d v_{n}=0$ and $\int x^{2} d v_{n} \leqq \int x^{2} d \nu$. Choose $\mu_{n}$ such that $\psi\left(\mu_{n}\right)=\nu_{n}, \mu_{n}(R)=\infty$ and $E\left\{\tau^{\mu_{n}}\right\}<\infty$. It is well known that $E\left\{\tau^{\mu_{n}}\right\}=\int x^{2} d \nu_{n} \leqq \int x^{2} d \nu$. As in Theorem 16, we can argue that $\left\{\mu_{n}\right\}$ has a convergent subsequence converging to some $\mu \in M^{*}$ and $\psi(\mu)=\nu$. Moreover along this subsequence $\tau^{\mu_{n}} \rightarrow \tau^{\mu}$ so by Fatou's lemma $E\left\{\tau^{\mu}\right\} \leqq \lim \inf E\left\{\tau^{\mu_{n}}\right\} \leqq \int x^{2} d \nu$.

A random variable is in the domain of normal attraction of the normal distribution if and only if it has a finite variance. Proposition 17, and the fact that for a Brownian motion stopping time $T, E\{T\}=E\left\{X_{T}^{2}\right\}$ if $E\{T\}<\infty$, gives us the result that a random variable $Y$ with mean zero can be realized as $X\left(\tau^{\mu}\right)$ for some $\mu \in M^{*}$ such that $E\left\{\tau^{\mu}\right\}<\infty$ if and only if $Y$ is in the domain of normal attraction of the normal distribution. For stable processes other than Brownian motion this statement is not true. We can say the following.

Proposition 18. Let $\left(X_{t}, \mathscr{F}_{t}\right)$ be a symmetric stable process of index $\alpha>1$. If a random variable $Y$ has the same distribution as $X\left(\tau^{\mu}\right)$ for some $\mu \in M^{*}$ where $E\left\{\tau^{\mu}\right\}$ $=a<\infty$, then $Y$ is in the domain of normal attraction of the distribution of $X_{a}$.

Proof. Let $X_{t}^{(n)}=n^{1 / \alpha} X(t / n)$ and $\mathscr{G}_{t}=\mathscr{F}(t / n)$. Then $\left(X_{t}^{(n)}, \mathscr{G}_{t}\right)$ is also a symmetric stable process of index $\alpha$. Let $T_{0}^{(n)}=0$. Define inductively $T_{i}^{(n)}$ to be $\tau^{\mu}$ for the symmetric stable process of index $\alpha$,

$$
X^{(n)}\left(t+\sum_{j=1}^{i-1} T_{j}^{(n)}\right)-X^{(n)}\left(\sum_{j=1}^{i-1} T_{j}^{(n)}\right)
$$

Then the random variables $T_{i}^{(n)}$ are identically distributed and, for fixed $n$, the $T_{i}^{(n)}$ are independent. But this implies that the random variables $S_{n}=(1 / n) \sum_{i=1}^{n} T_{i}^{(n)}$ converge in measure to $a$, since $E\left\{T_{i}^{(n)}\right\}=a$. Now $\sum_{i=1}^{n} T_{i}^{(n)}$ is a $\mathscr{G}_{l}$ stopping time so

$$
\left\{\sum_{i=1}^{n} T_{i}^{(n)} \leqq n t\right\} \in \mathscr{G}(n t)=\mathscr{F}(n t / n)=\mathscr{F}_{t}
$$


Thus $(1 / n) \sum_{i=1}^{n} T_{i}^{(n)}$ is an $\mathscr{F}_{t}$ stopping time. Since $(1 / n) \sum_{i=1}^{n} T_{i}^{(n)}$ converges to $a$ in probability, it follows that $X\left((1 / n) \sum_{i=1}^{n} T_{i}^{(n)}\right)$ converges to $X_{a}$ in distribution. But

$$
X\left((1 / n) \sum_{i=1}^{n} T_{i}^{(n)}\right)=n^{-1 / \alpha} X^{(n)}\left(\sum_{i=1}^{n} T_{i}^{(n)}\right)
$$

which has the same distribution as

$$
n^{-1 / \alpha}\left\{\sum_{i=1}^{n}\left[X^{(n)}\left(\sum_{j=1}^{i} T_{j}^{(n)}\right)-X^{(n)}\left(\sum_{j=1}^{i-1} T_{j}^{(n)}\right)\right]\right\} \text {. }
$$

But for each $n$ the random variables. $X^{(n)}\left(\sum_{j=1}^{i} T_{j}^{(n)}\right)-X^{(n)}\left(\sum_{j=1}^{i-1} T_{j}^{(n)}\right)$ are independent and have the same distribution as $Y$. Thus if $Y_{1}, Y_{2}, \ldots$ are independent random variables each having the same distribution as $Y$, then $n^{-1 / \alpha} \sum_{i=1}^{n} Y_{i}$ converges in distribution to $X_{a}$.

The converse of this theorem is not true if $\alpha<2$. The problem arises from the fact that being in the domain of normal attraction of $X_{1}$, say, depends only on the tails of the distribution, while being realized with a stopping time with finite expectation depends also on the "middle" of the distribution. Thus if one defines for some fixed $a>0$

$$
\begin{aligned}
Y(\omega) & =X_{1}(\omega) & & \text { if }\left|X_{1}(\omega)\right|>a, \\
& =a & & \text { if } 0 \leqq X_{1}(\omega) \leqq a, \\
& =-a & & \text { if }-a \leqq X_{1}(\omega)<0,
\end{aligned}
$$

then $Y$ is in the domain of normal attraction of $X_{1}$.

Now it follows from Proposition 18 that if $Y$ has the same distribution as $X\left(\tau^{\mu}\right)$ for some $\mu \in M^{*}$ such that $E\left\{\tau^{\mu}\right\}<\infty$, then $E\left\{\tau^{\mu}\right\}=1$. On the other hand, $\tau^{\mu}$ is clearly greater than $T_{A}$ where $A=\{x:|x|>a\}$. Since $E\left\{T_{A}\right\}>1$ for sufficiently large $a$, we can conclude, for these $a$, that if $X\left(\tau^{\mu}\right)$ has the same distribution as $Y$ then $E\left\{\tau^{\mu}\right\}=\infty$. The problem is an interesting one in view of the following proposition which says that some random variables in the domain of normal attraction of a symmetric stable distribution can be used to approximate the symmetric stable process.

Proposition 19. Suppose that $\left(X_{t}, \mathscr{F}_{t}\right)$ is a symmetric stable process of index $\alpha>1$. Let $Y_{1}, Y_{2}, \ldots$ be independent random variables each having the same distribution as $X\left(\tau^{\mu}\right)$ for $\mu \in M^{*}$ where $E\left\{\tau^{\mu}\right\}=1$. Define a sequence of processes

$$
Y_{n}(t)=n^{-1 / \alpha} \sum_{i=1}^{[n t]} Y_{i}, \quad 0 \leqq t \leqq 1 .
$$

Then each $Y_{n}(t)$ is equivalent to a process $V_{n}(t)$ defined on the same space as $X_{t}$ and, moreover, if $d$ is Skorokhod's $J_{1}$ metric then $d\left(X_{t}, V_{n}(t)\right)$ converges to zero in probability.

Proof. As in Proposition 18 we define the processes $\left(X_{t}^{(n)}, \mathscr{G}_{t}\right)$ and random variables $T_{i}^{(n)}$. Define

$$
V_{n}(t)=n^{-1 / \alpha} X^{(n)}\left(\sum_{i=1}^{[n t]} T_{i}^{(n)}\right), \quad 0 \leqq t \leqq 1 .
$$


In view of the choice of the $T_{i}^{(n)}$ and the independent increments, it is clear that $Y_{n}(t)$ is equivalent to $V_{n}(t)$.

Now $n^{-1 / \alpha} X^{(n)}\left(\sum_{i=1}^{n t]} T_{i}^{(n)}\right)=X\left(n^{-1} \sum_{i=1}^{[n t]} T_{i}^{(n)}\right)$ and $\sup _{0 \leqq t \leqq 1}\left|n^{-1} \sum_{i=1}^{[n t]} T_{i}^{(n)}-t\right|$ converges to zero in probability. (See, for instance, [10, Theorem 2.4].) Thus to complete the proof we need only show that if $\sup _{0 \leqq t \leqq 1}\left|n^{-1} \sum_{i=1}^{[n t]} T_{i}^{(n)}(\omega)-t\right|$ converges to zero then $d\left(X_{t}(\omega), X\left(n^{-1} \sum_{i=1}^{[n t]} T_{i}^{(n)}\right)(\omega)\right)$ also converges to zero. For convenience denote $n^{-1} \sum_{i=1}^{[n t]} T_{i}^{(n)}$ by $\gamma_{n}(t)$. Given $\varepsilon>0$ choose $0=t_{0}<t_{1}<\cdots$ $<t_{m-1}<1<t_{m}$ such that $\sup _{t_{i} \leqq t<t_{i+1}}\left|X_{t}-X_{t_{i}}\right|<\varepsilon / 2$ for $i=0,1, \ldots, m-1$. Let $\delta=\frac{1}{3} \min \left\{\varepsilon,\left(t_{i}-t_{i-1}\right),\left(1-t_{m-1}\right),\left(t_{m}-1\right)\right\}$ and suppose $\sup _{0 \leqq t \leqq 1}\left|\gamma_{n}(t)-t\right|<\delta$. Define for $i<m$

$$
\lambda\left(t_{i}\right)=\inf \left\{t: t_{i} \leqq \gamma_{n}(t)<t_{i+1}\right\} .
$$

Since for $t_{i}+\delta<t<t_{i+1}-\delta, t_{i}<\gamma_{n}(t)<t_{i+1}$ and $\gamma_{n}$ is nondecreasing, $\lambda\left(t_{i}\right)$ is well defined and $\lambda\left(t_{i}\right)<\lambda\left(t_{j}\right)$ if $i<j$. Since $\gamma_{n}$ is right continuous $t_{i} \leqq \gamma_{n}\left(\lambda\left(t_{i}\right)\right)<t_{i+1}$. Define $\lambda\left(t_{m}\right)=1$ and for $t_{i}<t<t_{i+1}$ define

$$
\lambda(t)=\frac{t_{i+1}-t}{t_{i+1}-t_{i}} \lambda\left(t_{i}\right)+\frac{t-t_{i}}{t_{i+1}-t_{i}} \lambda\left(t_{i+1}\right) .
$$

Then $\lambda$ is a homeomorphism. Moreover one verifies that $|\lambda(t)-t|<\delta \leqq \varepsilon$ and if $t_{i} \leqq t<t_{i+1}$ then $t_{i} \leqq \gamma_{n}(\lambda(t))<t_{i+1}$ so that $\left|X\left(\gamma_{n}(\lambda(t))\right)-X(t)\right|<\varepsilon$. Since $\varepsilon$ is arbitrary the theorem is proved.

An example of a distribution to which Proposition 19 is applicable is the distribution of $X_{T}$ where $T$ is the hitting time of the complement of any interval $[a, b]$ where $a<0<b$. Indeed if $\mu[a, b]=0$ but $\mu(\{x\})=\infty$ if $x \notin[a, b]$ then $\tau^{\mu}=T$.

\section{BIBLIOGRAPHY}

1. P. Billingsley, Convergence of probability measures, Wiley, New York, 1968. MR 38 \#1718.

2. R. M. Blumenthal and R. K. Getoor, Markov processes and potential theory, Academic Press, New York, 1968.

3. R. M. Blumenthal, R. K. Getoor and D. B. Ray, On the distribution of first hits for the symmetric stable processes, Trans. Amer. Math. Soc. 99 (1961), 540-554. MR 23 \#A4179.

4. L. E. Dubins, On a theorem of Skorohod, Ann. Math. Statist. 39 (1968), 2094-2097. MR 38 \#2837.

5: R. K. Getoor, Continuous additive functionals of a Markov process with applications to processes with independent increments, J. Math. Anal. Appl. 13 (1966), 132-153. MR 32 \#3125.

6. R. M. Loynes, Stopping times on Brownian motion: Some properties of Root's construction, Z. Wahrscheinlichkeitstheorie und Verw. Gebiete 16 (1970), 211-218.

7. H. McKean, Jr., Sample functions of stable processes, Ann. of Math. (2) 61 (1955), 564-579. MR 16, 1036.

8. P. A. Meyer, Probability and potentials, Blaisdell, Waltham, Mass., 1966. MR 34 \#5119.

9. I. Namioka, On certain onto maps, Canad. J. Math. 14 (1962), 461-466. MR 25 \#3343.

10. D. H. Root, Construction of almost surely convergent random processes, Thesis, University of Washington, Seattle, Wash., 1968.

11. A. V. Skorokhod, Studies in the theory of random processes, Addison-Wesley, Reading, Mass., 1965. MR 32 \#3082b.

Department of Mathematics, Dartmouth College, Hanover, New Hampshire 03755

Current address: Department of Mathematics, University of Hawaii, Honolulu, Hawaii 96822 